In Situ

Revue des patrimoines
In Situ

Revue des patrimoines

$42 \mid 2020$

Imagerie numérique et patrimoine culturel :

représentation et transmission des connaissances

\title{
Geste, matière et numérique
}

3D et archéologie du geste

Gestures, matter and digital tools: 3D and archaeology of actions

\section{Maud Mulliez}

\section{(2) OpenEdition \\ Journals}

Édition électronique

URL : http://journals.openedition.org/insitu/28367

DOI : 10.4000/insitu.28367

ISSN : $1630-7305$

Éditeur

Ministère de la Culture

Référence électronique

Maud Mulliez, "Geste, matière et numérique », In Situ [En ligne], 42 | 2020, mis en ligne le 12 juin 2020, consulté le 13 janvier 2021. URL : http://journals.openedition.org/insitu/28367 ; DOI : https://doi.org/ 10.4000/insitu.28367

Ce document a été généré automatiquement le 13 janvier 2021.

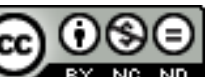

In Situ Revues des patrimoines est mis à disposition selon les termes de la licence Creative Commons Attribution - Pas d'Utilisation Commerciale - Pas de Modification 4.0 International. 


\title{
Geste, matière et numérique
}

\author{
3D et archéologie du geste \\ Gestures, matter and digital tools: 3D and archaeology of actions
}

\section{Maud Mulliez}

1 Devenue presque incontournable dans le domaine de l'archéologie, l'imagerie numérique 3D offre il est vrai de très nombreux champs d'application, du relevé de l'existant à la restitution, en passant par la modélisation d'hypothèses ou les systèmes d'information géographiques (SIG). L'usage de la 3D ne fournit donc pas seulement un outil de valorisation séduisant, même s'il contribue aussi de manière efficace et pédagogique à la diffusion de connaissances : la 3D est un véritable outil heuristique.

\section{Archéologie et histoire de l'art : 3D et expérimentation matérielle}

\section{D et recherche en histoire de l'art et archéologie}

\section{Relevé et observation}

2 L'imagerie 3D permet notamment de relever l'existant avec une très grande précision, grâce à la photogrammétrie ou à l'imagerie laser'1. Dans le cas d'objets fragiles ou difficiles d'accès, le bénéfice est évident : après acquisition, il est possible d'observer un objet sans le toucher et sous toutes ses faces si le modèle a pu être réalisé dans son entièreté. Il devient par ailleurs accessible, de n'importe où, sous la forme d'un fichier qui peut même être dupliqué afin de permettre à plusieurs personnes d'y travailler simultanément... en toute ubiquité. L'intérêt de la numérisation ne s'arrête pas là, loin s'en faut. Les potentialités offertes par les logiciels de visualisation pour faire varier l'éclairage ou modifier la texture, ainsi que la possibilité de réaliser des fac-similés par prototypage rapide sont des atouts majeurs. Lors de la restitution de la frise du Trésor des Marseillais à Delphes ${ }^{2}$, réalisée en collaboration avec le MAP-Gamsau ${ }^{3}$, les archéologues de l'université d'Aix-Marseille, le musée d'Archéologie méditerranéenne de Marseille, le musée de Delphes et l'Éphorie de Delphes, nous avons eu l'occasion de 
prendre la mesure de ces fabuleuses ressources. La gageure du projet consistait à remettre en situation un ensemble de fragments sculptés épars (nous disposions d'une vingtaine de fragments de quelques centimètres - ou dizaines de centimètres - pour une frise qui devait mesurer 29 mètres de longueur et présenter entre 100 et 140 personnages 4 ) : l'objectif premier était de leur donner une lisibilité suffisante pour le grand public, dans le cadre de l'exposition «Le Trésor des Marseillais. 500 av. J.-C., l'éclat de Marseille à Delphes" présentée en 2013 à la Vieille Charité, musée d'Archéologie méditerranéenne, à Marseille, qui était l'un des événements de l'année "Marseille-Provence capitale européenne de la culture ${ }^{5}$ ». Mais très vite, il nous est apparu que la nécessité d'aboutir à une restitution permettait du même coup de mieux appréhender certains objets en cherchant des indices pour les faire figurer de manière appropriée dans un ensemble continu et cohérent - ce à quoi une simple exposition juxtaposée des fragments n'aurait jamais donné lieu. Leur aspect particulièrement lacunaire, corrodé et épaufré en rendait la lecture très délicate : le moindre détail détecté pouvait donc s'avérer essentiel pour proposer un positionnement adéquat. Chacun d'eux a d'abord été numérisé lors d'une campagne d'investigation et d'observation menée sur place, à Delphes, dans les réserves du musée où se trouvaient la plupart des fragments et au sein du musée, pour les quelques éléments - les plus lisibles - exposés en vitrine. Par comparaison avec d'autres édifices analogues ${ }^{6}$, et du fait que les scènes sculptées d'époque archaïque répondaient à des schémas suffisamment codifiés pour permettre des correspondances fiables, la plupart des vestiges ont pu être placés dans une séquence cohérente. Quelques-uns en revanche résistaient... et il aura fallu les côtoyer longuement, les observer régulièrement pour pouvoir y déceler les caractéristiques nécessaires à leur identification.

Figure 1
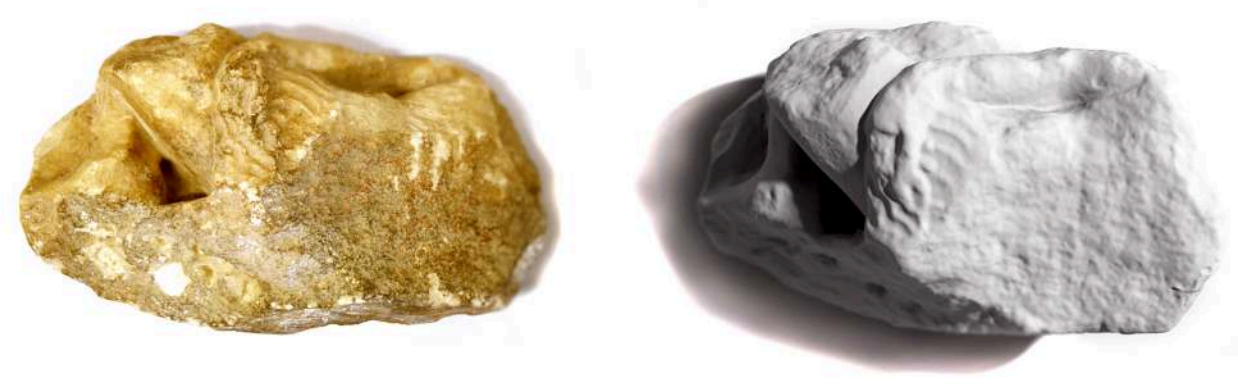

Fragment de la frise du Trésor des Marseillais à Delphes, conservé au musée de Delphes (inv. 5731).

Corps allongé avec un bras ramené sur la cuisse droite relevée. À l'arrière, le pied d'un autre personnage écrase la cuisse gauche tendue. À gauche : photographie du fragment. À droite: acquisition 3D sans texture permettant de mieux identifier le sujet.

Image : Maud Mulliez.

3 C'est là que les deux formes de rendus offertes par la numérisation, d'une part l'objet numérique visualisable dans un logiciel tel que Meshlab, et de l'autre l'impression 3D physique par prototypage rapide, ont démontré leur grande efficacité. Ainsi par exemple a-t-on pu s'apercevoir que les vestiges d'un corps allongé, qui aurait pu à l'origine faire penser à un personnage de banquet du fait de la position lascive de l'avant-bras sur la hanche, étaient en fait ceux d'une figure piétinée par un autre 
personnage, ce qui dès lors en faisait plutôt une victime dans une scène guerrière [fig. 1]. Un autre fragment représentait une tête particulièrement énigmatique qui pouvait à première vue sembler celle d'un personnage féminin grimaçant et montrant des dents proéminentes à l'excès : grâce à la visualisation 3D et à la modification artificielle de l'éclairage, notamment en lumière rasante, il s'est finalement avéré être un personnage masculin dont la joue était barrée d'une très légère trace figurant la limite d'une barbe $\mathrm{p}^{7}$. À l'arrière du casque, les restes d'un bouclier permettent d'en déduire la position sur la frise [fig. 2] ${ }^{8}$. C'est notamment grâce à la possibilité d'éliminer certains "artefacts» (terme pris ici dans le sens d'«accidents optiques») par la suppression de la texture correspondant à l'état actuel des vestiges couverts de concrétions que ces fines observations ont pu être menées. Or elles modifient considérablement l'analyse des fragments.

Figure 2
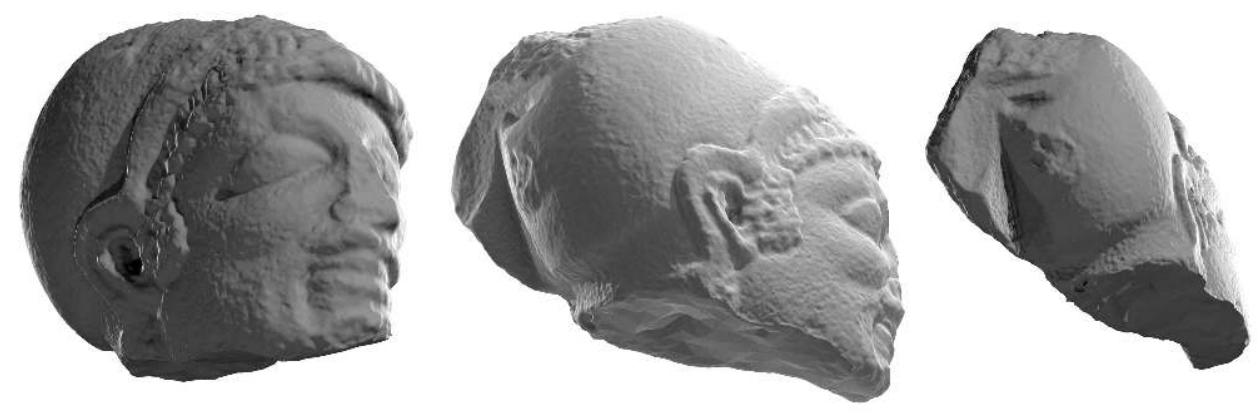

Visualisation du modèle 3D d'un fragment sculpté du Trésor des

Marseillais à Delphes représentant une tête de guerrier casqué, conservé au musée de Delphes (inv. 4314-3).

Image : L. Gügi, M. Vincitore.

\section{Hypothèses : restitution numérique et reconstitution physique}

L'imagerie numérique permet également de mettre en œuvre et de visualiser une ou plusieurs hypothèses de restitution qui, outre leur intérêt démonstratif, sont aussi un véritable outil de réflexion, souple et sans danger pour l'original. Il est rare en effet que le chercheur, si expert soit-il, soit en mesure de proposer d'emblée au spécialiste de la 3D une vision précise de tous les détails à restituer : il a en général une idée qui lui paraît assez claire, mais qui ne résiste pas entièrement à l'épreuve de la « mise en 3D ». Les questions que soulève la modélisation lui permettent donc de réfléchir à nouveau sur le sujet même de son étude, de le voir autrement, sous un autre angle, avec plus d'acuité ou avec de nouvelles interrogations. Mesures précises, forme d'un nez, profil d'une corniche, etc. : rien ne peut être laissé dans l'indécision ou dans l'imprécision. Il faut trancher ou proposer plusieurs hypothèses.

Lors de la restitution du Trésor des Marseillais, tant pour l'édifice entier que pour la frise sculptée déjà évoquée, de nombreux allers-retours ont été nécessaires entre les architectes du MAP, à qui il manquait parfois des données pour établir la maquette virtuelle, et les archéologues qui, grâce aux questions des architectes retournaient à 
leurs sources afin d'en tirer de nouvelles observations et de nouvelles conclusions. Ce n'est qu'au prix d'une série d'échanges sous la forme de descriptions, de schémas, de croquis, de modèles tirés de publications, de propositions amendées, de réunions collectives qu'il a été possible d'aboutir à la restitution finalement proposée [fig. 3].

Figure 3

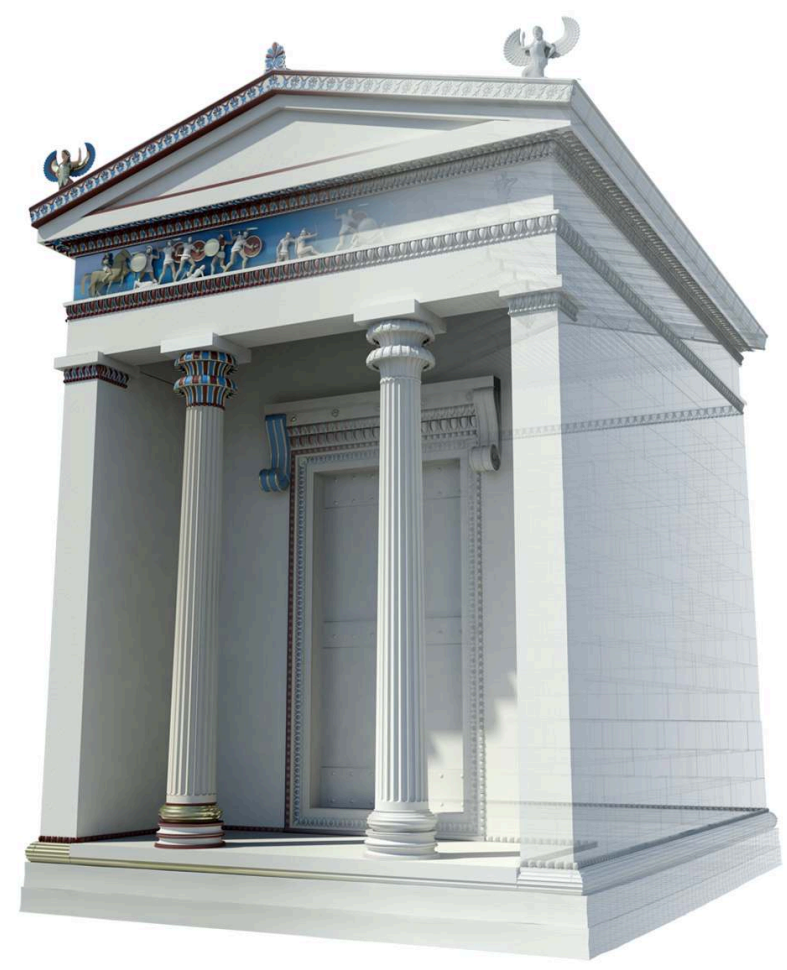

Restitution du Trésor des Marseillais, de la construction filaire à la mise en couleurs. Image : P. Desbottes, L. Gügi, M. Mulliez, M. Vincitore / cliché : MAP.

6 Le programme ANR Vesuvia9 , porté par Alexandra Dardenay, souligne ce même intérêt du travail de modélisation 3D qui permet et oblige tout à la fois une réflexion plus poussée permettant de mieux comprendre les enjeux : Alexandra Dardenay témoigne de ce processus au sujet de la restitution 3D de la maison de Neptune et Amphitrite à Herculanum ${ }^{10}$, dont il sera question plus loin au sujet du décor peint du triclinium. 
Figure 4

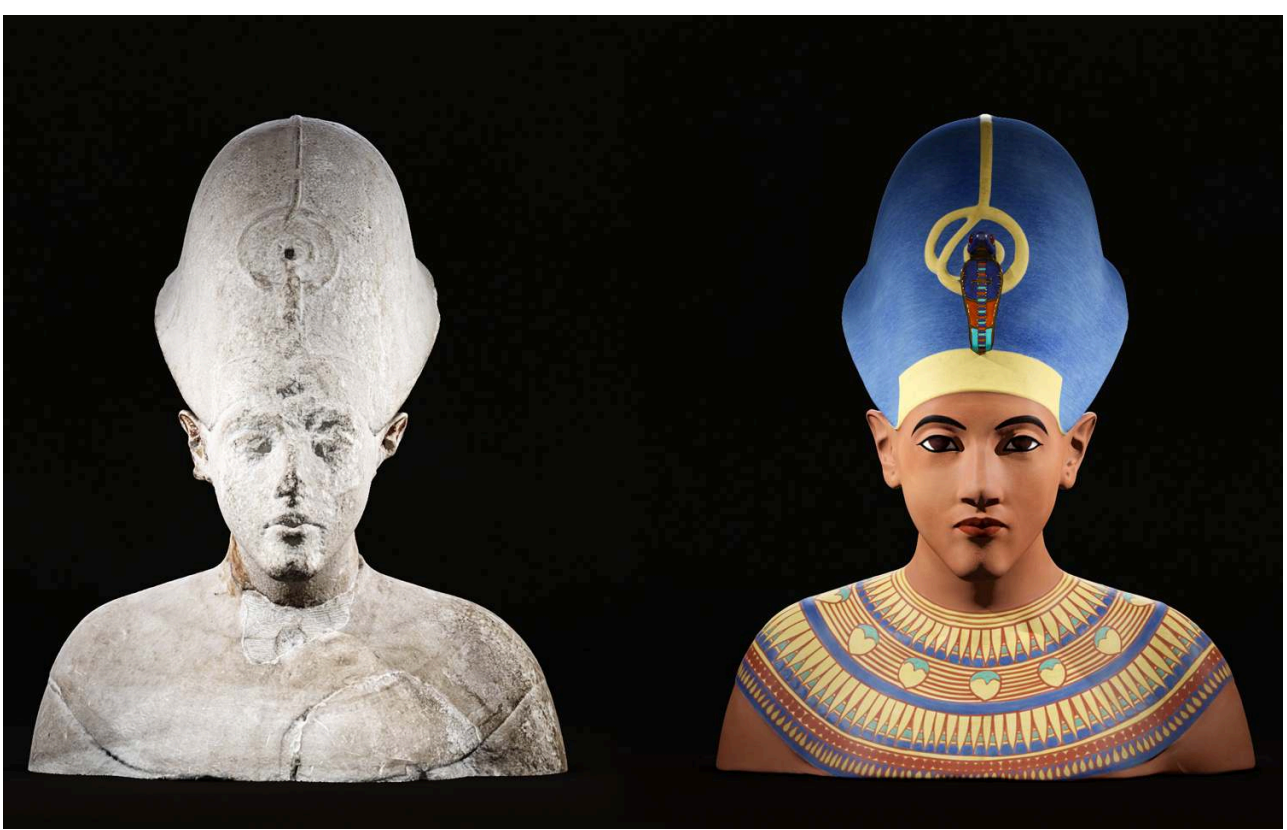

Buste d'Akhénaton conservé au Louvre (inv. E 11076). À gauche : numérisation de l'état actuel. À droite : restitution volumique et polychrome.

Image : D. Laboury, M. Mulliez, F. Daniel / cliché : Archeovision, Archeotransfert.

7 La restitution volumétrique et polychrome du buste d'Akhénaton conservé au Louvre (inv.E 11076), effectuée dans le cadre du programme Retrocolor 3 ${ }^{11}$ porté par l'UMS 3657 Archeovision et financé par la région Nouvelle-Aquitaine et l'université Bordeaux Montaigne, permet également d'illustrer ces échanges à double sens très féconds. Plusieurs traces de couleur subsistent encore à la surface du buste, mais elles sont très usées et se confondent par endroit avec des concrétions. Ainsi, au niveau du large collier du roi, dont la forme et la succession des éléments sont relativement établis, on devine certaines formes de motifs successifs qui paraissent presque " évidents » par rapport aux modèles analogues connus. Ce n'est qu'à l'épreuve de la mise en œuvre de la peinture virtuelle du modèle 3D que l'on s'est aperçu que l'alignement ne correspondait pas: il s'agit en fait d'une usure du bleu égyptien, pigment particulièrement granuleux, qui "par hasard" avait produit un réseau régulier de traces trompeuses. Il a donc fallu reprendre l'observation et revoir toute la structure du collier pour qu'il soit à la fois cohérent avec la connaissance théorique et les traces justes identifiées sur le buste. Les dessins préparatoires 2D que nous avions produit lors de nos allers-retours avec Dimitri Laboury, spécialiste de la période amarnienne, qui se limitaient à une vue de face, une vue de profil et des schémas aplatis, laissaient passer les imprécisions, autorisaient les raccourcis et les projections d'images créées dans nos cerveaux. Autant d'imprécisions que la 3D ne souffre pas [fig.4]. Ainsi, nous a confié Dimitri Laboury, le travail de colorisation et de modélisation 3D lui a permis de mieux appréhender des objets qu'il pensait pourtant connaître dans leurs moindres détails ${ }^{12}$. Le modèle 3D permet un véritable travail collaboratif où chacun parle de ce qu'il voit et non de ce qu'il a en tête, éventuellement déformé.

8 On mesure également les bénéfices de ce processus d'échanges fructueux pour les projets d'archéologie expérimentale ou de reconstitution matérielle : le fin connaisseur 
théorique d'un sujet s'aperçoit, grâce à la mise en œuvre réelle, que certains détails lui échappaient. Plusieurs expériences ont ainsi été tentées dans le cadre de l'exposition «Rituels grecs ${ }^{13}$ » au musée Saint-Raymond, musée d'archéologie de Toulouse: teintures, tissages, huiles parfumées, réalisation d'une stèle funéraire. Ces expériences ont été l'occasion pour Adeline Grand-Clément, qui en était à l'initiative, de revisiter des objets qu'elle connaissait pourtant mieux que quiconque ${ }^{14}$. Comment se terminent les coussins de banquet, lui demande-t-on, par des nœuds, des franges? Quelle couleur doit être utilisée en fil de chaîne et laquelle en fil de trame? Et elle, de retourner observer les vases bien connus pour y observer les finitions et donner à la tisserande, Zoë Montagu ${ }^{15}$, les indications justes. Même type de questionnements autour de la stèle sculptée et peinte pour l'occasion [fig. 5] : comment étaient réalisés les contours des motifs décoratifs ou figurés ? Étaient-ils tracés en amont, avant « remplissage », ou les figures étaient-elles cernées après application des champs colorés ? Les fonds étaientils laissés en réserve? Y avait-il des couches de préparation? Quels pigments, quels liants étaient utilisés? Autant de questions qui ne peuvent être laissées au hasard et nécessitent de se réinterroger avec un degré d'engagement supplémentaire: les réponses ne resteront pas théoriques, mais seront passées à l'épreuve de la réalité. La peinture tient-elle sur son support en marbre ? Et que se passerait-il si on la laissait en extérieur comme c'était le cas des stèles antiques ${ }^{16}$ ?

Figure 5
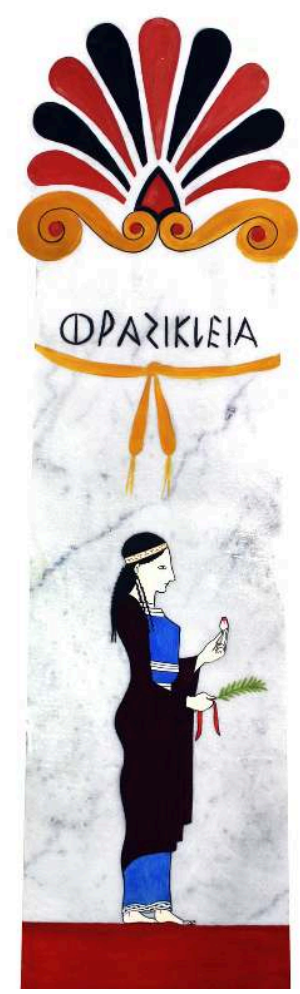

Stèle peinte créée dans un but expérimental et visuel à l'occasion de l'exposition " Rituels grecs » au musée Saint-Raymond de Toulouse (24 novembre $2017-25$ mars 2018), par François Grand-Clément et Maud Mulliez.

Image : Adeline Grand-Clément. 


\section{Matières et geste expérimental au service de la recherche et des restitutions numériques 3D}

L'outil numérique, si riche soit-il, est d'autant plus efficace qu'il ne se dissocie pas de la matérialité réelle, a fortiori s'il s'en fait une alliée. La restitution virtuelle suppose que l'infographiste ait des modèles à copier : or les matériaux qu'il doit reproduire ne sont plus toujours disponibles. L'archéologie expérimentale permet alors de recréer l'apparence de ces matériaux à partir des données disponibles et peut alors, en plus de l'intérêt intrinsèque d'une démarche expérimentale ${ }^{17}$, servir de ressource graphique pour la restitution.

Le programme Retro-Color 3D, déjà évoqué, a précisément été déposé pour répondre à une question jusque-là en suspens : comment traiter la couleur dans les restitutions numériques. Il s'agissait donc d'un programme transversal, délibérément diachronique, destiné à tester et développer une ou plusieurs méthodologies pour une restitution polychrome d'objets archéologiques qui soit à la fois scientifiquement rigoureuse et fidèle à un rendu visuel convaincant. Trois objets d'étude ont été choisis pour cela : le buste d'Akhénaton, le décor peint du triclinium 7 de la maison de Neptune et Amphitrite à Herculanum, tous deux déjà mentionnés, et l'ange porteur de lune du tympan du portail royal de la cathédrale Saint-André de Bordeaux. Des mesures colorimétriques sur les vestiges eux-mêmes peuvent être pertinentes, mais elles ne sont pas toujours possibles en raison de l'état de conservation des œuvres et doivent être considérées avec précaution car les couleurs qui subsistent peuvent avoir subi des transformations : il s'agit donc de bien sélectionner les zones pour faire ces mesures. Dans notre cas, ces mesures n'ont été possibles que sur les peintures romaines d'Herculanum; dans les deux autres cas, la peinture a presque totalement disparu, il n'en subsiste que d'infimes témoignages. Par ailleurs les mesures colorimétriques donnent des informations très utiles mais ne prennent en compte ni l'indice de réflexion (brillance, matité) ni les microreliefs nécessairement produits par l'application artisanale des couleurs sur un mur ou sur une sculpture. Or, une démarche expérimentale permet de dépasser ces deux limites : reproduire le processus de mise en œuvre originel permet d'obtenir un rendu de surface plausible correspondant à l'état de surface de l'objet au moment de sa production, sans les altérations et avec les effets de matière correspondant au processus mis en œuvre. 
Figure 6

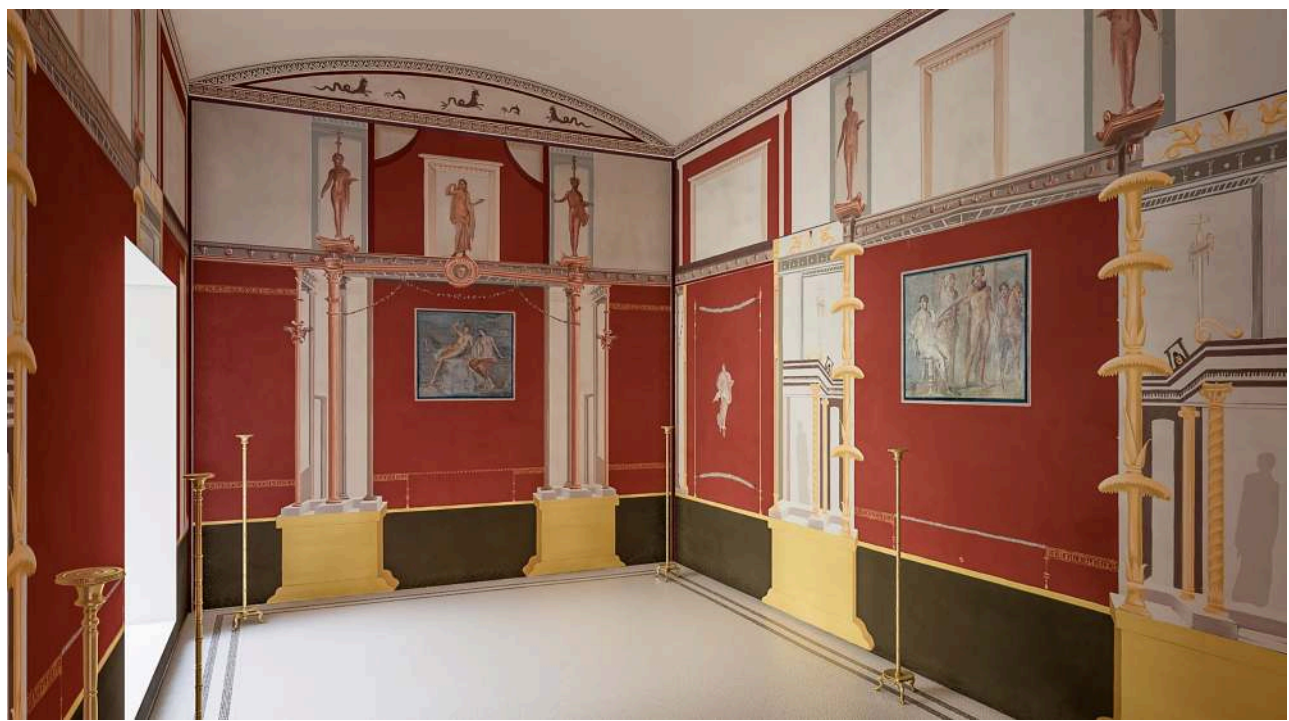

Restitution numérique du triclinium 7 de la maison de Neptune et Amphitrite, à Herculanum, réalisée dans le cadre du programme ANR Vesuvia en partenariat avec le programme Retro-Color 3D.

Image : Maud Mulliez / cliché : Vesuvia, Archeovision, Archeotransfert.

Dans le cas des peintures d'Herculanum, nous avons pu utiliser les nombreuses données accumulées lors de la recréation d'une fresque pour le musée Saint-Raymond de Toulouse, opération pour laquelle nous avions accumulé une large documentation photographique et vidéo ${ }^{18}$. La peinture virtuelle a été réalisée à l'aide de Photoshop, des calques de bump permettant de jouer sur les effets de matière [fig. 6]. Pour le buste d'Akhénaton, nous avons travaillé à partir des expérimentations réalisées par Hugues Tavier, chercheur-restaurateur qui fabrique lui-même son bleu égyptien, retrouve les compositions, utilise des pinceaux en chanvre ou en tige de roseau mâchonné, etc. ${ }^{19}$ : la peinture numérique du buste a été réalisée sur le logiciel Mudbox d'Autodesk [fig. 4]. Nous avons veillé à rendre les différents effets de matière liés aux différents pigments. Le bleu égyptien, très granuleux, produit d'importantes épaisseurs, très visibles en lumière rasante, alors que les pigments ocres, rouges ou jaunes, beaucoup plus miscibles et couvrants, produisent un rendu lisse; on peut aussi noter des effets de glacis qui apparaissent au niveau de la carnation, très visibles sur le buste de Nefertiti de Berlin, issu du même atelier. L'attention portée à ces effets de matière, grâce à une démarche expérimentale parallèle, permet de donner une idée de la polychromie beaucoup plus juste mais aussi plus acceptable pour nos mentalités habituées à la patine des couleurs qui va souvent jusqu'à l'effacement. 


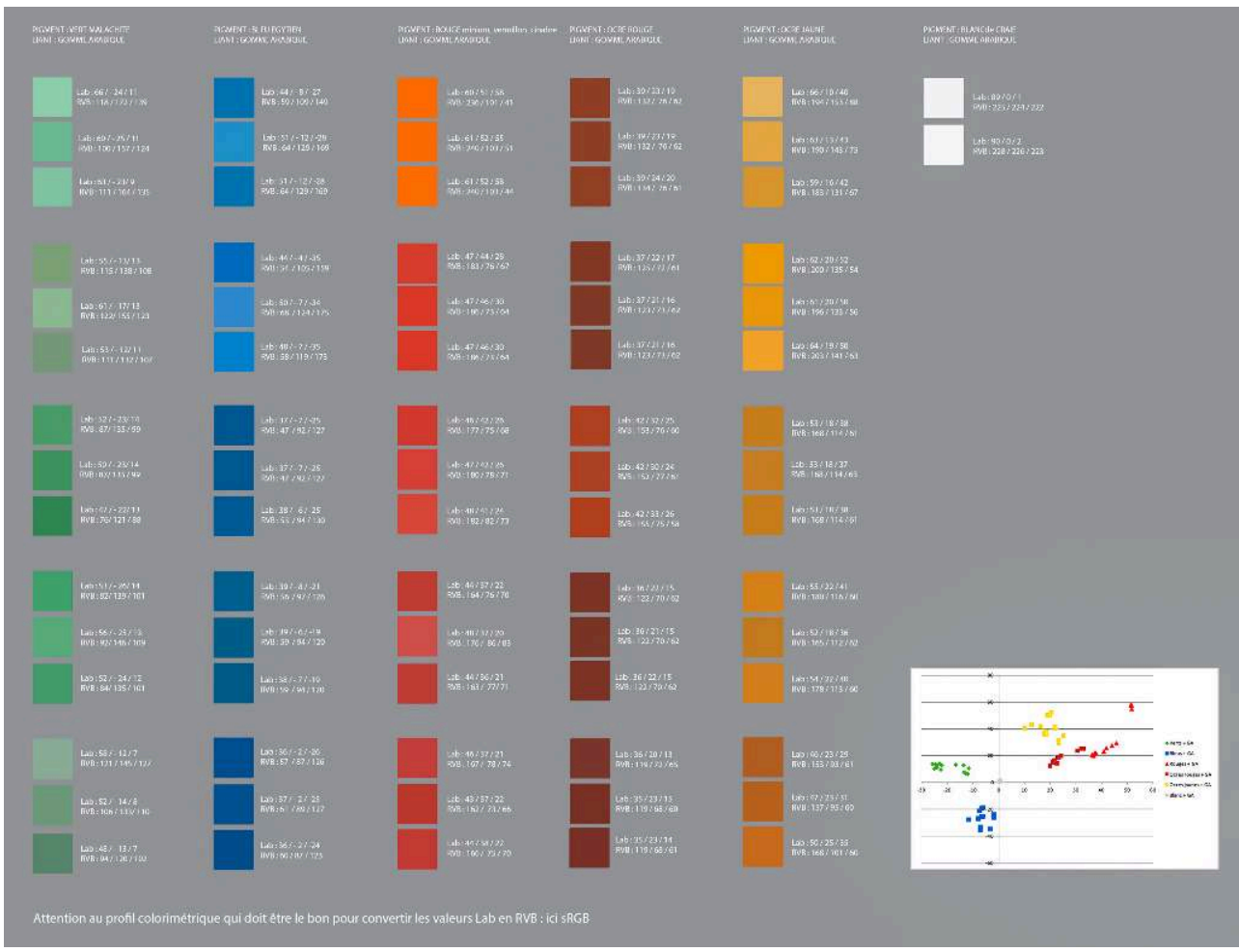

Palette virtuelle obtenue par mesures colorimétriques à partir des expérimentations réalisées sur la base des analyses physicochimiques des couleurs du tympan de la cathédrale Saint-André de Bordeaux.

Image : Maud Mulliez.

Enfin, pour la sculpture du tympan de la cathédrale de Bordeaux, des analyses physicochimiques ${ }^{20}$ nous ayant permis de reproduire la stratigraphie picturale, nous avons réalisé un échantillonnage de couleurs en prenant pour un pigment donné toute la gamme colorée offerte et en testant les différents liants possibles. Des mesures colorimétriques nous ont alors permis de créer une palette virtuelle [fig. 7], les effets de matière étant reproduits à partir du panneau expérimental réel. La peinture virtuelle de l'ange a également été réalisée sur le logiciel Mudbox d'Autodesk. En dehors des visages et de la carnation, les couleurs d'origine étaient vraisemblablement appliquées en aplat mais la couleur uniforme était posée à l'aide d'un pinceau, ce qui crée nécessairement des irrégularités de pose (contrairement à ce qu'on peut obtenir avec la peinture au pistolet, ou en numérique, par le remplissage uniforme d'une couleur donnée). Ainsi, dans notre modèle 3D, même les couleurs unies ont été appliquées à l'aide d'un pinceau virtuel (et non un remplissage d'une zone prédéfinie), opération qui nécessitait aussi de suivre la topographie de la sculpture, comme on le ferait pour peindre une sculpture réelle. Cette mise en œuvre, calquée sur le réel, permet d'obtenir un rendu à la fois plus crédible et plus conforme à la matérialité de l'objet [fig. 8]. 

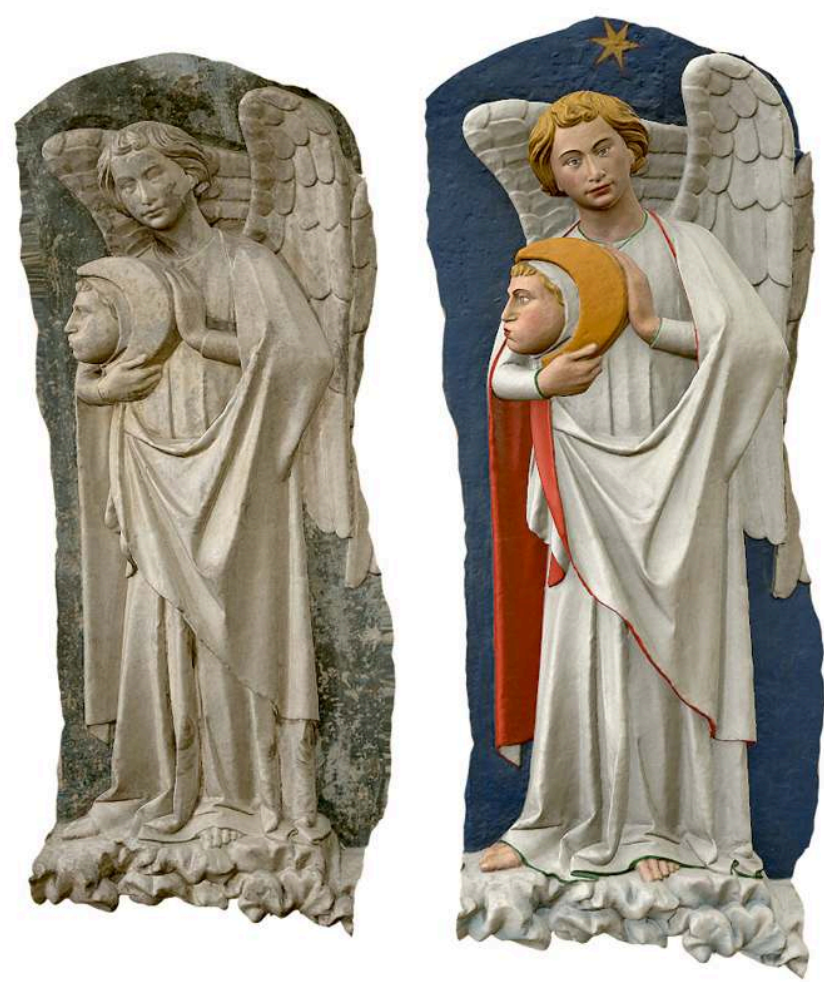

L'ange porteur de lune du tympan du portail royal de la cathédrale Saint-André à Bordeaux. À gauche : restitution numérique de l'état actuel. À droite : proposition de restitution polychrome.

Image : Maud Mulliez / cliché : Archeovision, Archeotransfert.

\section{Numérique et 3D : pour une archéologie du geste}

S'intéresser au geste artisanal ouvre d'autres perspectives d'échanges entre les nouvelles technologies, à travers l'usage du numérique et de la 3D, et une " autre forme d'archéologie ", celle qui étudie le patrimoine des gestes. Dans ce domaine, la chambre des métiers et de l'artisanat du Tarn-et-Garonne s'est montrée particulièrement précurseur avec son initiative de «Répertoire numérique du geste artisanal ${ }^{21}$ » et le programme "Artisan numérique ». Le projet « L'Empreinte du Geste », sélectionné par l'Idex de Bordeaux dans le cadre de son programme Arts et Sciences en $2017^{22}$, est dédié à cette recherche avec un double aspect : retrouver les gestes ayant abouti à un réseau de traces qui détermine une œuvre, d'une part, dans une démarche d'archéologie du geste, et d'autre part, dans une visée purement artistique, créer une nouvelle œuvre, qui serait la quintessence des gestes, détachés de l'œuvre qu'ils ont produite.

\section{Genèse du projet « L'Empreinte du Geste »}

Les vestiges archéologiques nous livrent à leur surface des traces d'un savoir-faire parfois facile à reconnaître, d'autres fois plus mystérieux: ainsi par exemple, les peintures murales romaines, dont le rendu lisse et brillant a toujours étonné les spécialistes de la fresque, portent-elles des traces incrustées dans la matière qui peuvent être des clefs de compréhension de leur processus de création. Tenter de 
retrouver, à partir d'une trace, le geste et l'outil qui ont permis de la réaliser, tel est le point de départ du projet "L'Empreinte du Geste » mené en collaboration avec l'Inria de Bordeaux et l'Institut d'optique d'Aquitaine sous la forme d'une résidence ${ }^{23}$. Pour établir le lien entre une trace et le geste qui l'a produite, il nous faut tout d'abord une base de données de traces et de gestes dont le rapport est bien établi puis, par apprentissage (de type intelligence artificielle), de nouveaux modèles pourront être générés. Avec «L'Empreinte du Geste ", nous sommes aux prémices de ces recherches et cherchons en premier lieu à établir et analyser ce lien en choisissant une technique artistique assez simple pour éviter d'avoir trop de paramètres à prendre en compte. Dans cette phase exploratoire, nous avons donc choisi de nous concentrer sur des œuvres réalisées à l'encre de Chine sur papier. Nous cherchons à établir le lien entre ces œuvres et les gestes de l'artiste grâce à l'enregistrement de ses mouvements à l'aide d'un ensemble de marqueurs utilisés avec un trio de caméras (OptiTrack) et la numérisation des traces produites sur le papier afin d'en extraire des corrélations.

Figure 9

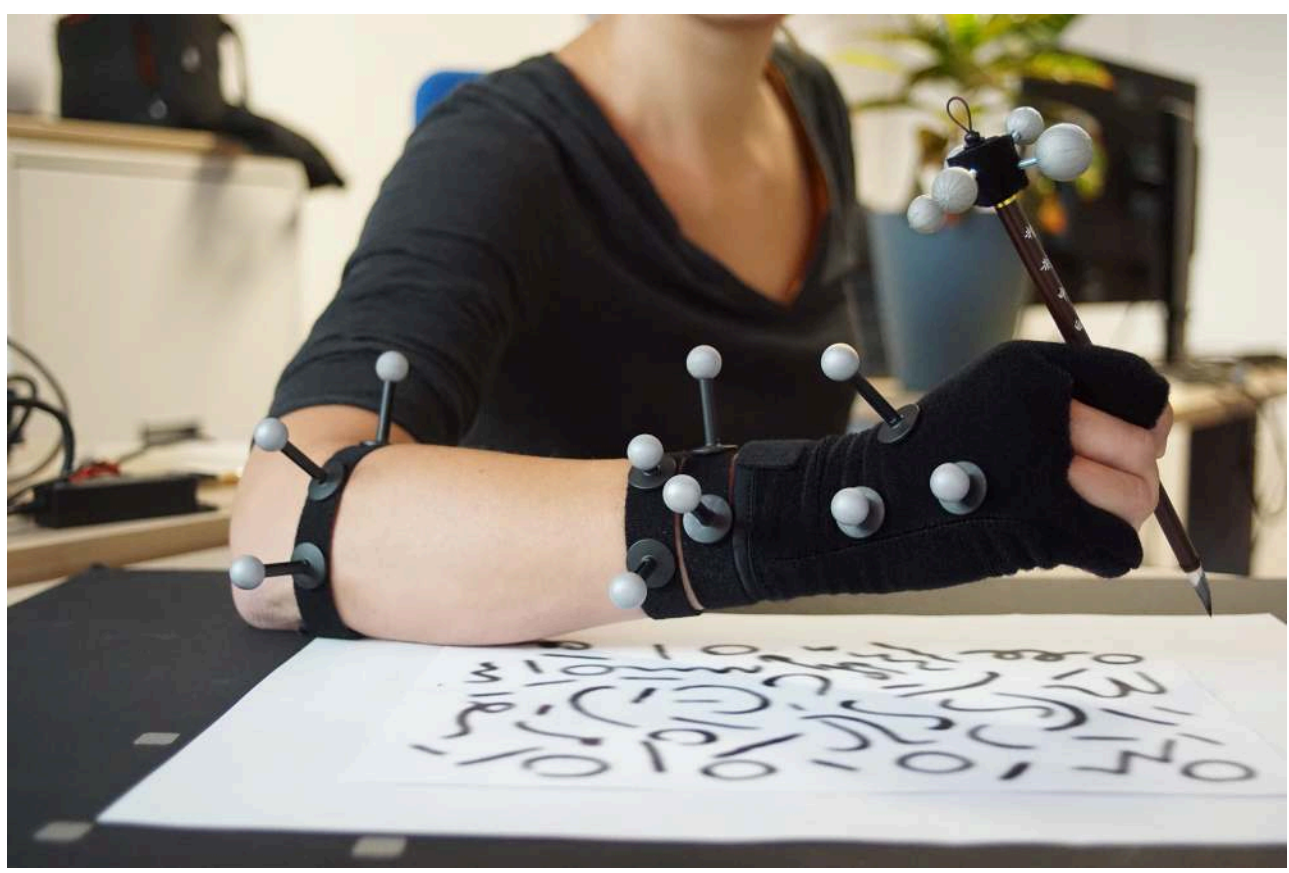

Disposition des marqueurs sur l'avant-bras, le poignet, la main et l'outil permettant d'enregistrer les mouvements.

Cliché : L'Empreinte du Geste.

Les marqueurs sont disposés à des endroits spécifiques sur l'avant-bras, le poignet, la main et le pinceau [fig. 9] : trois capteurs par point choisi permettent de constituer les rigid bodies, c'est-à-dire des corps rigides, non déformables mais mobiles en trois dimensions, dont on pourra suivre le parcours sur un graphique à trois axes; le plan de travail est également localisé. L'enregistrement des mouvements permet de visualiser la différence de trajectoires entre les quatre rigid bodies définis [fig. 10]. 
Figure 10

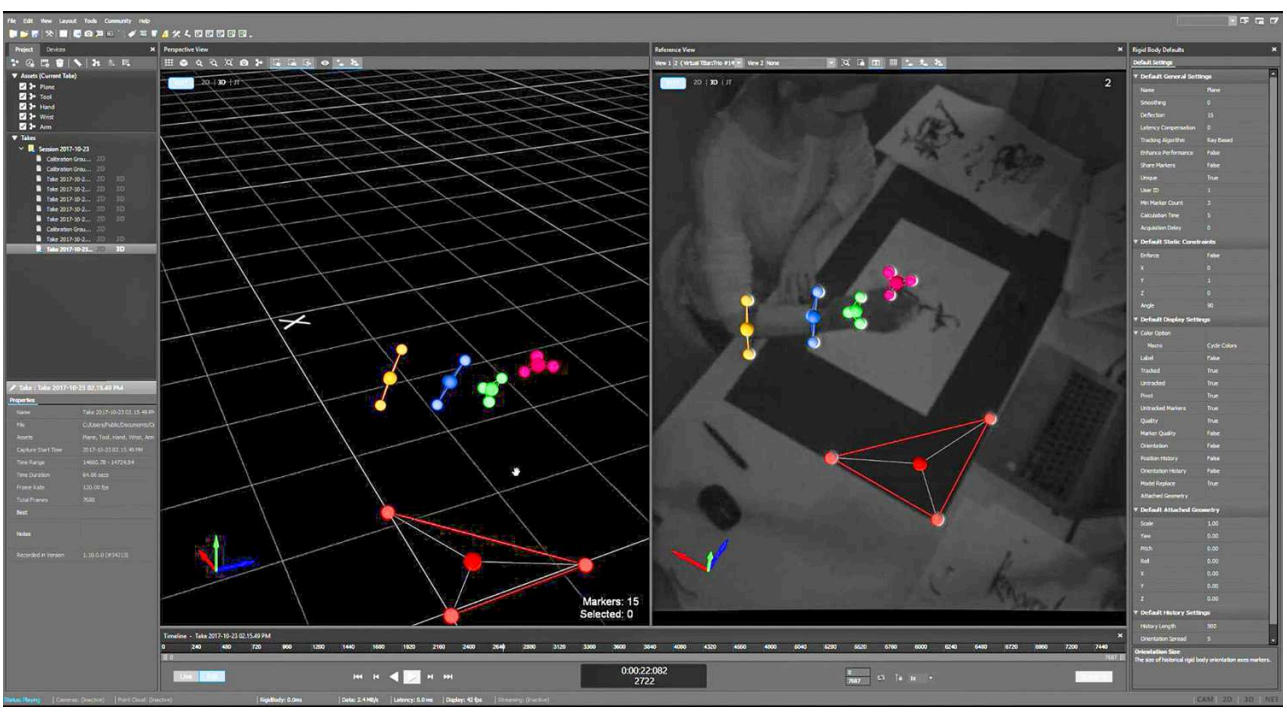

Visualisation des rigid bodies et des marqueurs du plan de travail à partir de deux des caméras du trio Optitrack qui permettent d'enregistrer les mouvements (capture d'écran).

Cliché : L'Empreinte du Geste.

Une première série de tests réalisés à partir de tracés prédéfinis a permis de vérifier que les caméras suivaient bien les mouvements des marqueurs, et de voir si l'on parvenait effectivement à établir le lien spatial entre les tracés scannés et les trajectoires des rigid bodies. Nous avons pu ensuite passer à des créations libres. Deux types de dessins ont été mis en œuvre : des croquis de modèles et de la calligraphie, éventuellement abstraite. Les deux univers produisent des gestes très différents: la calligraphie fait appel à des gestes d'une faible amplitude, relativement précis, tandis que les croquis de modèles, plus instinctifs, suscitent des gestes plus amples et rapides. Grâce à des algorithmes prenant en compte les trajectoires, la vitesse, l'orientation et la pression exercée sur le papier - par un calcul de distance entre le pinceau et le plan de travail -, des corrélations entre les gestes réalisés et l'œuvre ont pu être établies. Elles ont permis de "rejouer " la création de l'œuvre: le résultat de cette recréation est intéressant car il nous permet de vérifier la justesse de ces corrélations tout en montrant l'écart qui subsiste entre l'œuvre réelle et sa reproduction virtuelle [fig. 11]. 
Figure 11
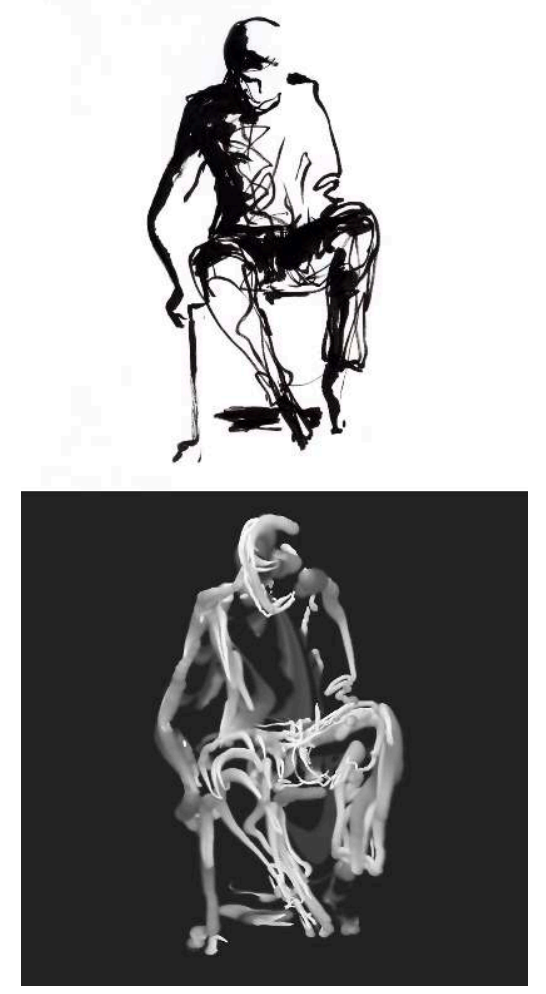

Croquis de dessin réalisé à l'encre de Chine sur papier, avec enregistrement des mouvements et sa reproduction virtuelle tenant compte de la trajectoire des mouvements enregistrés, de leur vitesse, de la pression et de l'orientation du pinceau.

Image : Maud Mulliez / cliché : L’Empreinte du Geste.

17 Les premiers travaux réalisés dans le cadre de cette résidence ont permis d'extraire les gestes réalisés, de les mettre en corrélation avec l'œuvre créée et de les visualiser sous différentes formes [fig. 12]. Ils ont été présentés à l'Espace 29 lors du festival Facts ${ }^{24}$ qui s'est tenu à Bordeaux du 14 au 24 novembre 2017. Une vidéo a été réalisée à cette occasion $^{25}$. 


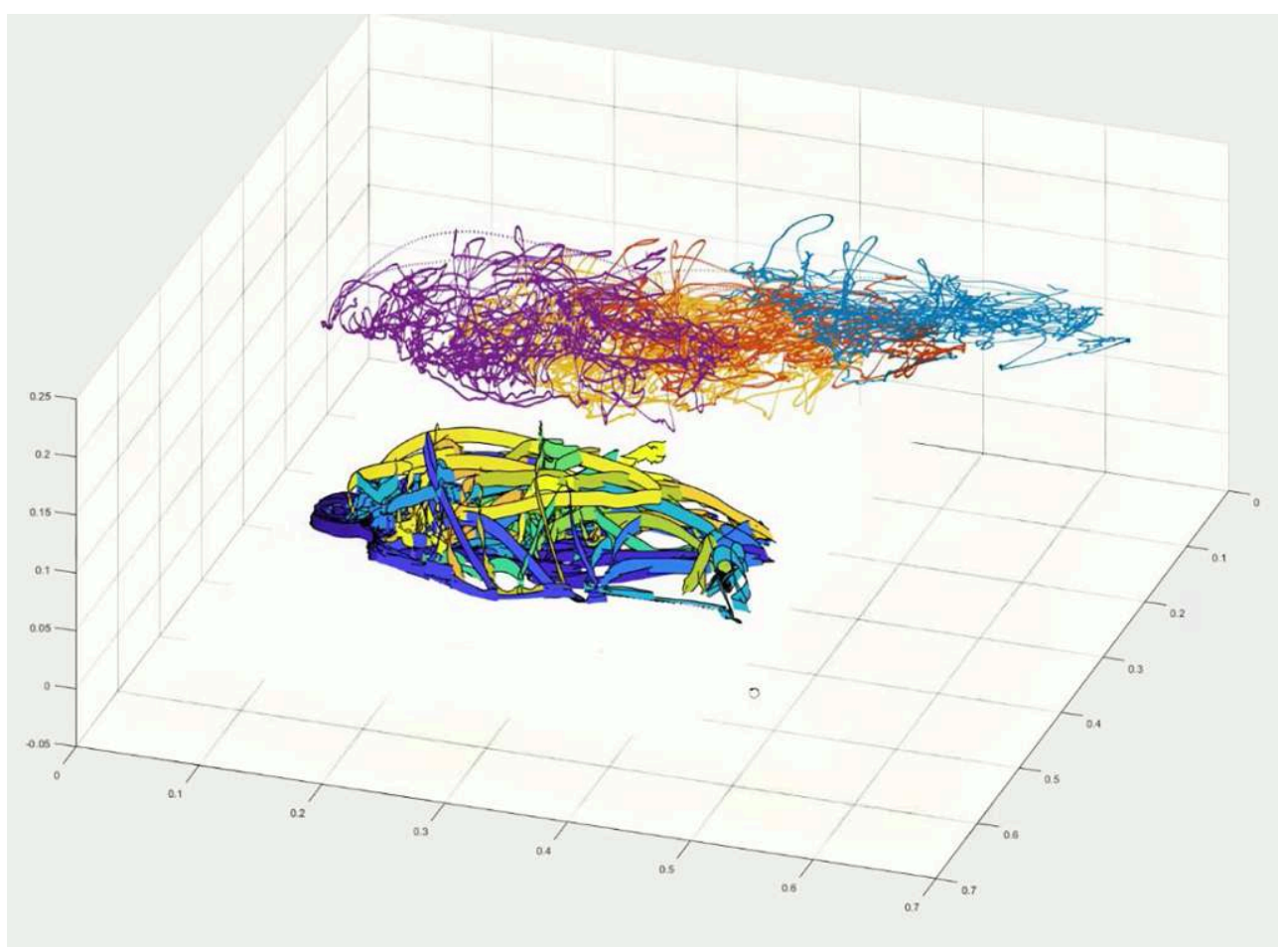

Capture d'écran permettant de visualiser la trajectoire des mouvements enregistrés superposée au dessin réalisé pendant l'enregistrement des mouvements.

Cliché : L’Empreinte du Geste.

\section{Vers une nouvelle œuvre}

Loin de chercher à remplacer l'artiste par une machine, notre projet artistique est au contraire de produire une nouvelle œuvre, uniquement extraite des gestes réalisés pour une création donnée, mais se détachant de cette création originelle. Il s'agit en quelque sorte de sa "sublimation ", au sens premier du terme - passage de l'état solide, concret, à l'état gazeux, abstrait-, de l'œuvre originale : ici le geste devient l'œuvre même. Cette phase créative dont nous avons présenté une forme pré-figurative [fig. 13] lors de l'exposition à l'Espace 29, puis plus aboutie et sous une forme interactive avec le public qui le souhaitait, lors de l'une des éditions de Station-Campus à Bordeaux, le 29 novembre $2019^{26}$, est toujours en cours de création. 


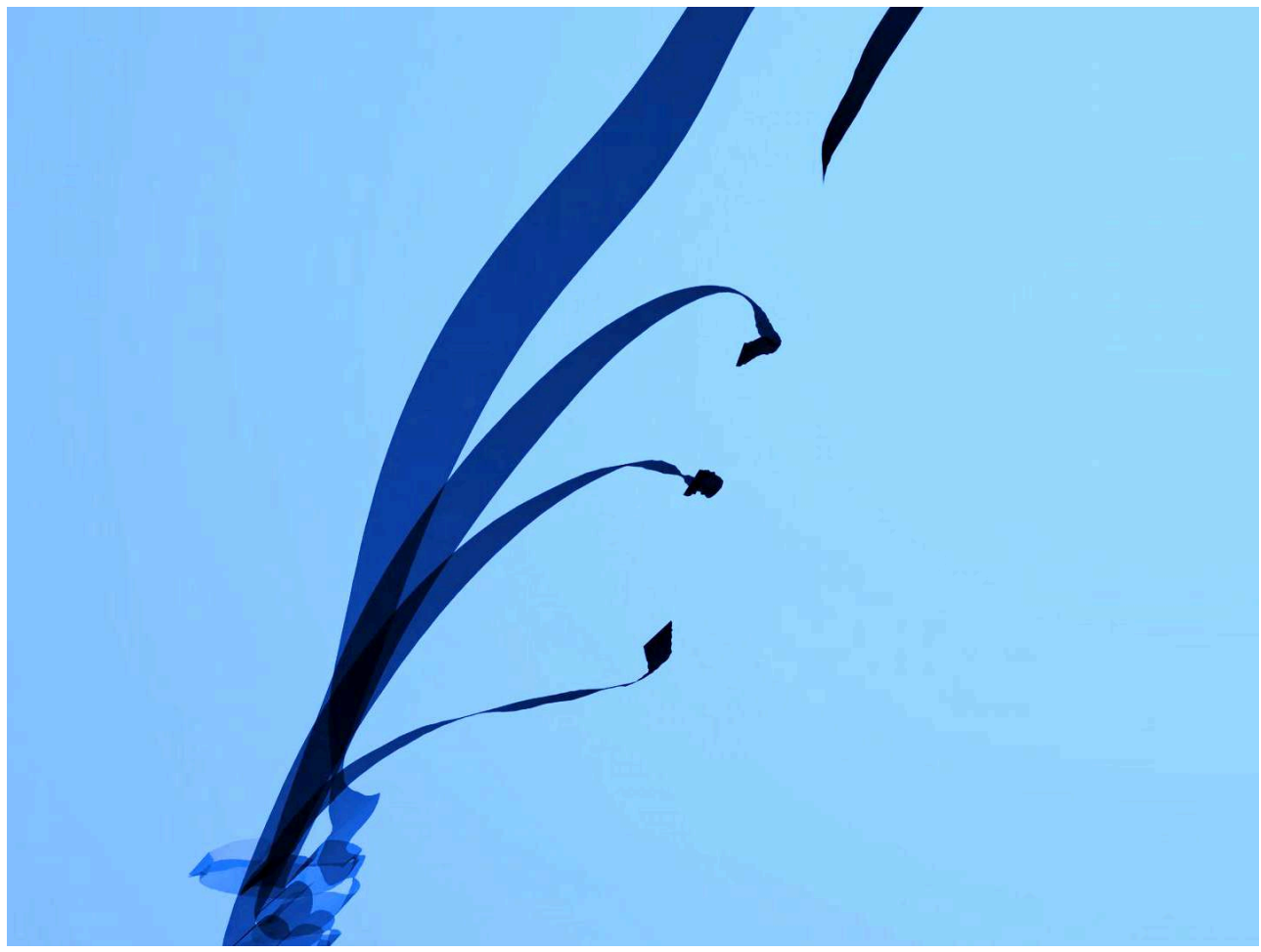

Arrêt sur image de la première ébauche de l'œuvre-geste en train d'être créée au sein du projet "L'Empreinte du Geste ».

Cliché : L'Empreinte du Geste.

\section{Conclusion}

19 L'engouement que connaissent les nouvelles technologies, et l'imagerie 3D en particulier, peut susciter la méfiance à l'égard d'images qui peuvent paraître trop séduisantes pour être scientifiques. Il est vrai qu'il n'est pas évident de relever le défi de concurrencer la qualité graphique produite par les studios de jeux vidéo ou de séries qui plongent le joueur ou le spectateur dans un univers devenu de plus en plus crédible et réaliste, et souvent assez bien documenté. Les archéologues, quant à eux, sont confrontés à des données souvent lacunaires et à la nécessité de garantir le contenu rigoureux de leurs restitutions. La prise en compte de la matérialité des objets qu'ils traitent, en passant éventuellement par une étape expérimentale dans leur processus de recherche, est l'une des clefs qui peut leur permettre à la fois d'assurer un contenu plus juste à leur modèle et de proposer un rendu satisfaisant tant pour le grand public que pour les chercheurs. L'usage de la 3D ne se limite d'ailleurs pas à la production de modèles utiles à la valorisation, mais, comme on l'a vu autour de plusieurs exemples, constitue un véritable outil de recherche qui ne souffre pas l'imprécision que notre cerveau peut, quant à lui, s'autoriser, et offre une matière qui facilite efficacement le travail collaboratif. Enfin, la captation et l'enregistrement des mouvements permettent de nouvelles perspectives à ce que l'on peut appeler l'archéologie du geste ${ }^{27}$ : cet autre usage de la 3D devrait permettre de corréler des traces archéologiques aux gestes et outils de leurs auteurs et ouvrir ainsi la voie d'un nouveau patrimoine : celui des gestes. 


\section{NOTES}

1. Sur les différents procédés et l'usage de la 3D en archéologie, voir, parmi une très large bibliographie : GILIGNY François \& VERGNIEUX Robert (dir.), Les Nouvelles de l'archéologie, $\mathrm{n}^{\circ} 146$, «La 3D en archéologie», 2016; DE LUCA Livio, La Photomodélisation architecturale. Relevé, modélisation, représentation d'édifices à partir de photographies, Paris, Eyrolles, 2009.

2. Dans les sanctuaires grecs, les trésors (thesauros ou oikos en grec) sont des édifices dont l'aspect s'apparente à celle d'un petit temple. Ils avaient la fonction d'abriter des offrandes mais étaient également en soi des offrandes. Le trésor des Marseillais fut édifié par les Massaliètes vers 500 avant notre ère dans le sanctuaire d'Athéna à Delphes.

3. MAP-Gamsau est l'équipe fondatrice de l'UMR 3495 MAP « Modèles et simulations pour l'architecture et le patrimoine » (CNRS / ministère le Culture / École nationale d'architecture de Marseille). «Gamsau » est l'acronyme du Groupe de recherche pour l'application des méthodes scientifiques à l'architecture et à l'urbanisme, basé sur le campus CNRS Joseph-Aiguier à Marseille.

4. D'après PICARD Charles \& LA COSTE-MESSELIÈRE Pierre de, "Les trésors ioniques ", Fouilles de Delphes, t. IV, Monuments figurés, fasc. 2, Sculpture, Paris, De Boccard, 1928, p. 53.

5. Voir GARSSON Muriel (dir.), Le Trésor des Marseillais. 500 av. J.-C., l'éclat de Marseille à Delphes, catalogue d'exposition (Marseille, musée d'Archéologie méditerranéenne, 12 janvier-15 avril 2013), Paris, Somogy, 2012 ; «Le trésor des Marseillais », Archéologia, hors-série, nº 15, 2013 ; MULLIEZ Maud, JOCKEY Philippe \& VINCITORE Mauro, « Virtual reconstruction and experimental attempt in archaeology. The Massalian treasury in Delphi », Proceedings of the 2013 Digital Heritage International Congress, Marseille, IEEE, 2013, p. 579-585.

6. En particulier le trésor de Siphnos et le trésor des Athéniens à Delphes et le temple d'Aphaia à Égine.

7. Cette trace de barbe avait déjà été décelée par Théophile Homolle au moment de la découverte: une «barbe courte [...] peinte et non détaillée au ciseau» (PICARD Charles \& LA COSTE MESSELIÈRE Pierre de, art. cit., p. 28, note 2). Elle justifie la présence d'une moustache au-dessus de la bouche, car il n'y a pas, à cette époque, de représentation de personnage moustachu sans barbe et explique aussi les trois bourrelets situés sous le nez, mieux que ne le permettait l'interprétation de dents proéminentes entre la lèvre inférieure et la lèvre supérieure. 8. Voir la frise proposée dans les différentes publications mentionnées en note 4. Voir également : http://restauration-peinture.eu/archeologie/le-tresor-des-marseillais-experience/

9. «Vivre ensemble. Société et urbanisme d'une ville de l'Italie antique»: https:// vesuvia.hypotheses.org/

10. Lors de sa communication donnée à la journée d'étude "Le bois dans l'architecture » (5-6 avril 2018) et parue dans la revue Pallas : DARDENAY Alexandra, « Le bois dans l'architecture domestique à Herculanum. Mise en œuvre de propositions de reconstruction 3D dans la Casa di Nettuno ed Anfitrite ", Pallas. Revue d'études antiques, nº 110, 2019, p. 245-256.

11. http://archeovision.cnrs.fr/retrocolor3d

12. C'est ainsi qu'il nous a proposé de titrer la publication de notre présentation lors du colloque «Reconstruction of polychromy / Restituer les couleurs", dont les actes sont sous presse : «Étude du buste d'Akhénaton du Musée du Louvre par sa restitution 3D polychrome », insistant ainsi sur la fonction active de la restitution dans le processus même de l'étude du buste: LABOURY Dimitri, MULLIEZ Maud \& DANIEL François. «Étude du buste d'Akhénaton du Musée du Louvre par sa restitution 3D polychrome", in MULLIEZ Maud (dir.). Reconstruction of Polychromy/ Restituer les couleurs, actes du colloque «Virtual Retrospect 2017 », Bordeaux, Ausonius, à paraître. 
13. Voir la présentation de l'exposition sur le site du musée (https://saintraymond.toulouse.fr/ Rituels-grecs-Une-experience-sensible_a1086.html) et dans une vidéo: https:// www.youtube.com/watch?time_continue $=12 \& \mathrm{v}=26 \mathrm{BfO} 7 \mathrm{plds0}$

14. Des précisions sur l'exposition, sa préparation et sur les expérimentations qu'elle a occasionnées sont disponibles sous la forme d'un carnet de recherches en ligne (http:// synaesthes.hypotheses.org/) ainsi que dans la publication du colloque déjà mentionné : GRANDCLÉMENT Adeline \& MULLIEZ Maud, «Rituels grecs en couleurs : les dessous chromatiques d'une exposition polysensorielle», in MULLIEZ Maud (dir.), Reconstruction of Polychromy, Restituer les couleurs, actes du colloque « Virtual Retrospect 2017 », Bordeaux, Ausonius, à paraître.

15. http://www.zoemontagu.com/

16. Nous avons d'ailleurs rencontré un problème avec le mélange choisi pour la carnation du personnage : contrairement aux autres couleurs, celle-ci, restée pulvérulente, n'a pas tenu. Nous avons donc procédé, en cours d'exposition, à une restauration de la stèle... La question de la tenue en extérieur reste à tester, ce qui donnera sans doute lieu à de nouveaux essais.

17. Voir par exemple les travaux réalisés autour de la fresque romaine : http://www.tectoriaromana.com/

18. Ibid.; AUSSILLOUX-CORREA Aude \& MULLIEZ Maud, « Re-création d'une fresque antique : une archéologie expérimentale », in DARDENAY Alexandra \& CAPUS Pascal, L'Empire de la couleur, catalogue d'exposition (musée Saint-Raymond-musée des Antiques de Toulouse, 15 nov. 201422 mars 2015), Toulouse, musée Saint-Raymond-musée des Antiques de Toulouse, 2014, p. 16-23 ; MULLIEZ Maud \& AUSSILLOUX-CORREA Aude, «La fresque antique : retrouver les gestes et les couleurs. Un exemple d'archéologie expérimentale », in BOISLEVÉ Julien, DARDENAY Alexandra \& MONIER Florence (dir.). Peintures et stucs d'époque romaine. Une archéologie du décor, Bordeaux, Ausonius, coll. «Pictor», 2016, p. 371-384; MULLIEZ Maud \& AUSSILLOUX-CORREA Aude, «La peinture murale antique : une gageure technique à l'épreuve de l'archéologie expérimentale », in CARRIVE Mathilde, Remployer, recycler, restaurer. Les autres vies des enduits peints, Rome, École française de Rome, 2017, p. 93-105, pl. XXVII-XXIX.

19. LABOURY Dimitri, MULLIEZ Maud \& DANIEL François, «Étude du buste d'Akhénaton du Musée du Louvre par sa restitution 3D polychrome", in MULLIEZ Maud, Reconstruction of Polychromy/ Restituer les couleurs, actes du colloque «Virtual Retrospect 2017 », Bordeaux, Ausonius, à paraître ; ainsi que, dans le même volume : TAVIER Hugues, «La reconstitution des techniques picturales de l'Égypte ancienne. Recherches expérimentales à propos de la Nécropole Thébaine ".

20. Voir les précisions dans MULLIEZ Maud, «La restitution polychrome de l'ange porteur de lune ", in Bordeaux, Saint-André, primatiale d'Aquitaine, La grâce d'une cathédrale, Strasbourg, La Nuée bleue, 2017, p. 286-287, n² 212.

21. Voir les différentes vidéos du Répertoire numérique du geste artisanal (RNGA) disponibles sur la chaîne Vimeo, parmi lesquelles : https://vimeo.com/134633525.

22. http://idex.u-bordeaux.fr/fr/n/Connexion-avec-la-societe/Arts-et-sciences/r3043.html 23. Résidence à l'Institut national de recherche en sciences du numérique (Inria). Ce projet est une collaboration entre Maud Mulliez, Pascal Barla, Pierre Bénard et Romain Pacanowski, équipe Manao (Inria, LaBRI, LP2N) : https://facts2017.u-bordeaux.fr/RESIDENCES/L-empreinte-dugeste.html

24. http://www.facts-bordeaux.fr/

25. http://restauration-peinture.eu/creation/lempreinte-du-geste/

26. https://www.u-bordeaux.fr/Evenements/De-la-vie-de-campus/Station-Campus-2-etape-5

27. Démarche initiée par Sophie Archambault de Beaune: ARCHAMBAULT DE BEAUNE Sophie, Pour une archéologie du geste. Broyer, moudre, piler. Des premiers chasseurs aux premiers agriculteurs, Paris, CNRS Éditions, 2000. 


\section{RÉSUMÉS}

L'imagerie 3D est devenue un outil essentiel dans le domaine de l'archéologie. Elle permet notamment de relever l'existant et de visualiser des hypothèses de restitution qui, outre leur intérêt démonstratif, nous aident aussi à progresser dans notre réflexion. Cet outil est d'autant plus efficace qu'il ne se dissocie pas de la matérialité, surtout s'il en fait son alliée. Nous présentons ici quelques exemples de l'intérêt de croiser l'expérience numérique avec la pratique matérielle. Nous y montrons combien l'oscillation entre l'un et l'autre est d'un grand bénéfice dans la recherche en histoire de l'art et archéologie; d'une part à travers la question de l'objet numérisé, donc dématérialisé, et son impression 3D, d'autre part autour de l'archéologie expérimentale qui permet de nourrir la précision des restitutions numériques.

Mais il est une autre archéologie, celle qui s'intéresse au patrimoine des gestes. Dans ce domaine aussi, numérisation et 3D sont des outils précieux. Le projet «L'Empreinte du Geste » sélectionné par l'Idex de Bordeaux dans le cadre de son programme Arts et Sciences est dédié à cette recherche. Tenter de retrouver à partir d'une trace le geste et l'outil qui ont permis de la réaliser, tel est le point de départ de ce projet mené grâce à une collaboration avec l'Inria de Bordeaux et l'Institut d'optique d'Aquitaine. Encore dans sa phase exploratoire, ce projet a pour but d'analyser le lien entre geste et trace grâce à l'enregistrement des mouvements de l'artiste à l'aide d'un ensemble de marqueurs (OptiTrack) et la numérisation des traces produites afin d'en extraire des corrélations.

3D imaging has become an essential tool in archaeology. Digital acquisition of remains and 3D restitution based on several hypotheses have both a demonstrative interest and a research goal. And it is as much efficient when it is not dissociated from materiality, but rather if it takes it into account. This paper presents some examples that illustrate the advantage of combining digital experience with practice. It shows how useful it is to combine digital and materiality in art history and archaeology using both digitisation of an object and its 3D printing, or using material experimentation to provide better results in digital reconstructions in sculpture and architecture.

Another kind of archaeology can be mentioned, one that deals with gesture. In this field, digitisation and 3D are also of particular interest. Our project "L'Empreinte du geste" selected by the IDEX of Bordeaux as part of its Arts and Sciences program is dedicated to this research. Trying to rediscover the gesture and tools that have produced it with the help of an archaeological trace: that was the starting point of the project, carried out in collaboration with the INRIA at Bordeaux and the Institut d'optique d'Aquitaine. Still in its exploratory phase, our proposal aims at analysing the correlations between gesture and traces thanks to a motion capture system (using a set of markers OptiTrack) and the digitisation of the piece of work created.

\section{INDEX}

Keywords : digital, digitisation, 3D, archaeology, experimentation, experimental archaeology, scientific imaging, restitution, virtual restoration

Mots-clés : numérique, numérisation, numérisation 3D, archéologie, archéologie expérimentale, imagerie scientifique, restitution, restauration virtuelle 


\section{AUTEUR}

\section{MAUD MULLIEZ}

Chercheure associée au laboratoire " Archéologies et sciences de l'Antiquité » (ArScAn, UMR 7041), et laboratoire Archéovision (UMS 3657, CNRS / Université Bordeaux Montaigne).

maud@maudmulliez.com 